\title{
Design and Implementation of Active Filter for Data Acquisition System
}

\author{
Ms.Mya Thandar Kyu, Dr.Zaw Min Aung and Dr.Zaw Min Naing
}

\begin{abstract}
A filter is a device that passes electric signals at certain frequency or frequency ranges while preventing the passage of others. This paper focuses on active low-pass Butterworth filters design. In communication, data acquisition system usually requires anti-aliasing low-pass filter as well as low-pass noise filter in their preceding signal condition stages. In this paper design of fourth-order low-pass filters is the main topic of consideration. This paper will examine how to implement Butterworth filter and design methods. And this paper describes mathematics calculations and implementation of Butterworth filter with MATLAB and Circuit Maker.
\end{abstract}

Key words: low-pass filter, implementation, active filter, Butterworth

\section{INTRODUCTION}

Analog filters are continues-time filters that can be implemented with resistors, capacitors, amplifiers or specialized elements, etc. as passive and active filters, which have been used for along time in electrical engineering. Analog filters can be found in almost every electronic circuit. Audio systems use them for preamplification, equalization, and tone control. In communication systems, filters are used for tuning in specific frequencies and eliminating others. Digital signal processing systems use filters to prevent the aliasing of out-of-band noise and interference. The data acquisition system signal chain that includes an analog filter is shown in Fig.1.

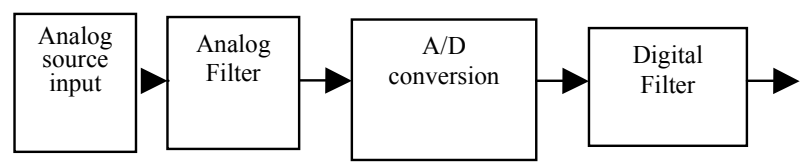

Fig .1. The data acquisition system signal chain can utilize analog or digital filtering techniques or combination of both.

Manuscript received December 10, 2008. This work was supported in part by the Ministry of Science and Technology, Union of Myanmar.

Ms. Mya Thandar Kyu is with the Mandalay Technological University, Mandalay, Myanmar. Contact Phone: 095-2-88704(Electronic Engineering Department), Fax: 095-2-88702(office, MTU), e-mail: myathandarkyu@gmail.com and myathandar@gmail.com. Dr. Zaw Min Aung and Dr.Zaw Min Naing are her supervisors and they are rector of Mandalay Technological University, Myanmar and pro-rector of Hlaing Tharyar Technological University, Myanmar
A system that includes an analog filter, a digital filter or both is shown in Figure 1. When an analog filter is implemented, it is done prior to the analog-to-digital conversion. In contrast, when a digital filter is implemented, it is done after the conversion from analog-to-digital has occurred. Analog filtering can remove noise superimposed on the analog signal before it reaches the Analog-to-Digital Converter. In these types of systems, an analog filter can reduce noise in the out-of-band frequency region. In the data acquisition system, the analog signal will go directly into an active low pass filter. In this system, the bandwidth of interest of the analog signal is DC to $1 \mathrm{kHz}$. The low pass filter will be designed so that high frequency signals from the analog input do not pass through to the A/D Converter in an attempt to eliminate aliasing errors. The implementation analog signal will go directly into an active low pass filter. The implementation and order of this filter will be modified according to the design parameters. Excluding the filtering function, the anti-aliasing filter will not modify the signal further that is implement a gain or invert the signal. The low pass filter segment will be followed by a 12-bit A/D Converter. The sampling rate of the $\mathrm{A} / \mathrm{D}$ Converter will be $20 \mathrm{kHz}$, making $1 / 2$ of Nyquist equal to $10 \mathrm{kHz}$. The ideal signal-to-noise ratio of a 12-bit A/D Converter of $74 \mathrm{~dB}$. This design parameter will be used when determining the order of the anti-aliasing filter.

In the second the section of this application, the anti-aliasing filter design and implementation will be performed. In this section anti-aliasing filter requirements will be determined. Filter characteristics and architecture will also be discussed in this section. Moreover, filter design consideration over Butterworth filter, Chebyshev type I filter and Bessel filter will be performed. Then, implementation with $4^{\text {th }}$ Butterworth low pass filter design will be carried out in order to prevent the aliasing parameter. The third and final part of this application, the comparison of the Circuit Maker simulation and calculated result will be discussed.

\section{ANTI-ALIASING FILTER}

These three design parameters will be used to implement appropriate anti-aliasing filters:

1. Cut-off frequency for filter must be $1 \mathrm{kHz}$ or higher.

2. Filter attenuates the signal to $-74 \mathrm{~dB}$ at $10 \mathrm{kHz}$.

3. Flat frequency response in the pass-band.

Analysis of Butterworth, Chebyshev type I and Bessel filter types, then should be done to decide which type is most suitable for implementation, depending on the system requirements. 


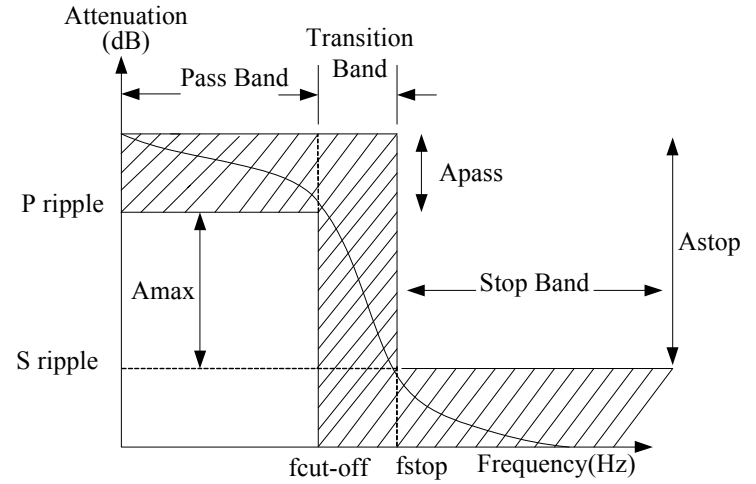

Fig.2.Analog low pass filter design parameters requirements

In Fig.2. $A_{\text {pass }}$ is the $3 \mathrm{~dB}$ limit, $A_{\text {stop }}$ is the attenuation requirement in the stop band, $\mathrm{f}_{\text {cut-off }}$ is the frequency end of pass band, set to $1 \mathrm{kHz}, \mathrm{f}_{\text {stop }}$ is the frequency at the beginning of stop band, the zone between $\mathrm{f}_{\text {pass }}$ and $\mathrm{f}_{\text {stop }}$ is the transition zone, $\mathrm{P}_{\text {ripple }}$ is the passband ripple, $\mathrm{S}_{\text {ripple }}$ is the stopband ripple and defined as the difference between the gain in the pass band region and the gain that is achieved in the stop band region is $\mathrm{A}_{\text {MAX }}=\mathrm{A}_{\mathrm{PASS}}-\mathrm{A}_{\mathrm{STOP}}$.

\section{FILTER CHARACTERISTIC AND ARCHITECTURE}

If an ideal low-pass filter existed, it would eliminate signals above the cutoff frequency, and perfectly pass signals below the cutoff frequency. In real filters, various trade-offs are made to get optimum performance for a given application.

Butterworth filters are termed maximally-flat-magnituderesponse filters, optimized for gain flatness in the pass-band and the attenuation is $-3 \mathrm{~dB}$ at the cutoff frequency. Above the cutoff frequency the attenuation is $-20 \mathrm{~dB} / \mathrm{decade} /$ order. The transient response of a Butterworth filter to a pulse input shows moderate overshoot and ringing.

Bessel filters are optimized for maximally-flat time delay (or constant-group delay). This means that they have linear phase response and excellent transient response to a pulse input. This comes at the expense of flatness in the pass-band and rate of rolloff. The cutoff frequency is defined as the 3$\mathrm{dB}$ point.

Chebyshev filters are designed to have ripple in the passband, but steeper rolloff after the cutoff frequency. Cutoff frequency is defined as the frequency at which the response falls below the ripple band. For a given filter order, a steeper cutoff can be achieved by allowing more pass-band ripple. The transient response of a Chebyshev filter to a pulse input shows more overshoot and ringing than a Butterworth filter.

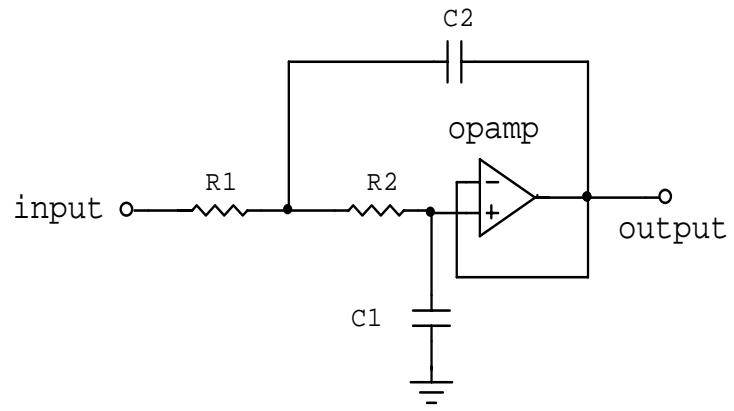

Fig.3.General architecture for Sallen-Key of a two-pole filter section

The architecture that has been used to implement the lowpass filter is called Sallen-Key. This was chosen because of its simplicity compared to other know architectures as multiple feedback and state variable, where the latter is for precision performance. A circuit diagram for Sallen-Key is shown in Fig.3. Sallen-Key architecture use to non-inverting port as input which normally is set for unity gain operation. This gives a very accurate unity gain filter in the pass-band. Pole-pair Q-value below there is specific good for SallenKey and only need one Op-amp to build a two-pole filter section.

\section{FILTER DESIGN CONSIDERATION}

The three filter types Butterworth, Chebyshev type I and Bessel that were described theoretically in the previous section, are going to be simulated in Math-lab in this section. The magnitude and phase function of each filter type are going to be presented in following subsection, will the requirement written in section (I). The result of following analysis decides which filter type that is going to be implemented.

\section{A. Butterworth filter}

Butterworth filter with its flat image in the pass-band and low steepness after the cutoff frequency of $1 \mathrm{kHz}$, requires being a $4^{\text {th }}$ order, to fulfill the attenuation requirement of $-74 \mathrm{~dB}$ at $10 \mathrm{kHz}$ see Fig.4. for filter response.

\section{B. Chebyshev type I filter}

Chebyshev type I filter with ripple of $1 \mathrm{~dB}$ in the passband and with steeper fall off rate after the cutoff frequency than Butterworth. It requires only being a $3^{\text {rd }}$ order, to be able to follow attenuation of $-74 \mathrm{~dB}$ at $10 \mathrm{kHz}$ see Fig.5. for the filter response.

\section{Bessel filter}

Bessel filter, $5^{\text {th }}$ order filter is needed to fulfill requirement of attenuation of $-74 \mathrm{~dB}$ from pass band region to $10 \mathrm{kHz}$ see Fig .6. for the filter response. 
Proceedings of the International MultiConference of Engineers and Computer Scientists 2009 Vol I

IMECS 2009, March 18 - 20, 2009, Hong Kong

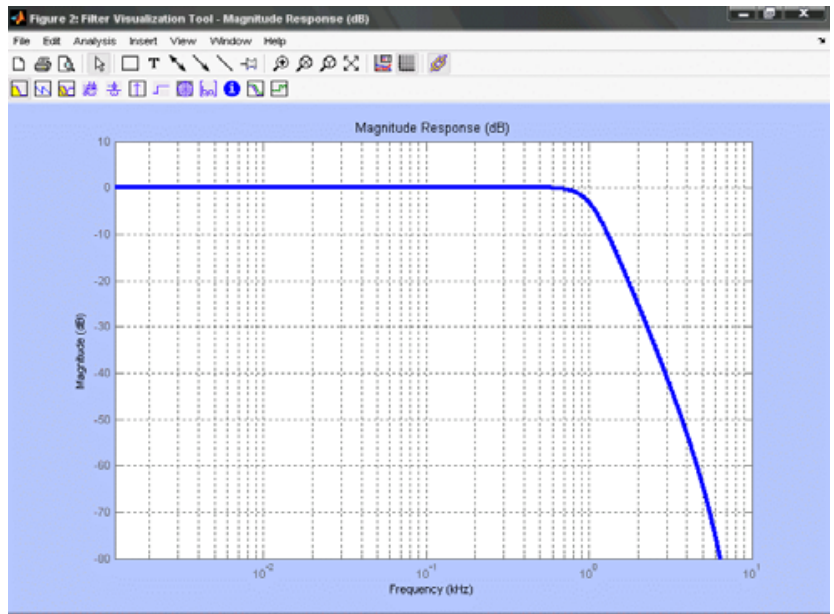

Fig.4. (a) simulated responses of $4^{\text {th }}$ order Butterworth lowpass filter frequency response in $\mathrm{dB}$

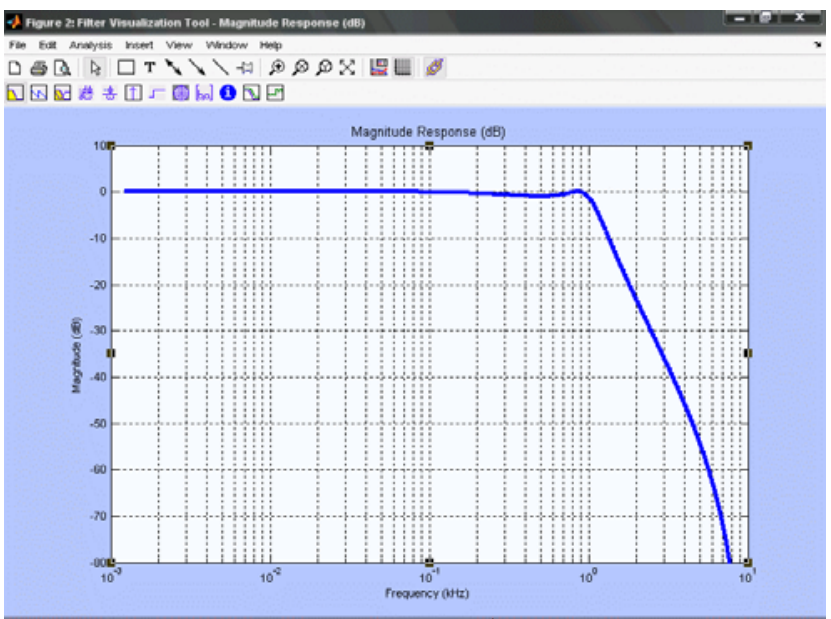

Fig.5. (a) simulated responses of $3^{\text {rd }}$ order Chebyshev type I low-pass filter frequency response in $\mathrm{dB}$

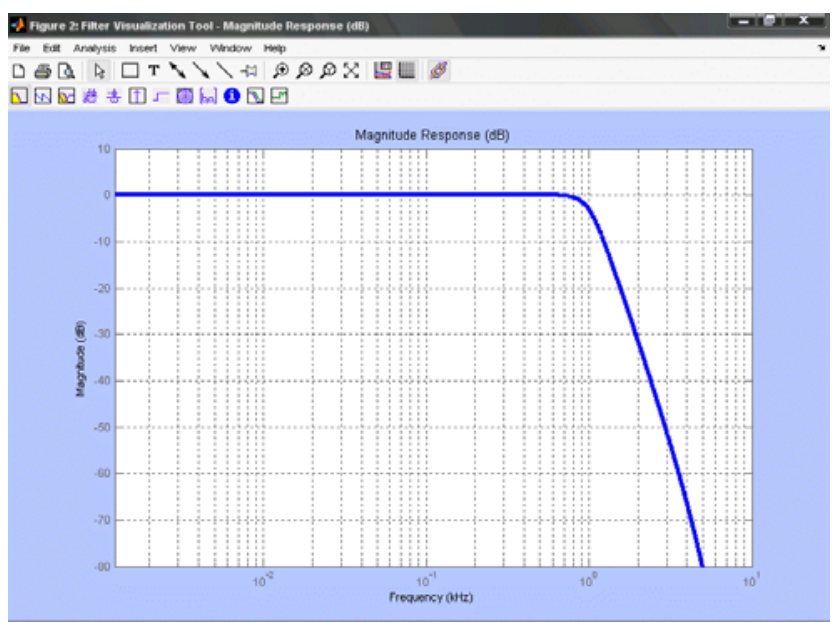

Fig.6. (a) simulated responses of $5^{\text {th }}$ order Bessel low-pass filter frequency response in $\mathrm{dB}$

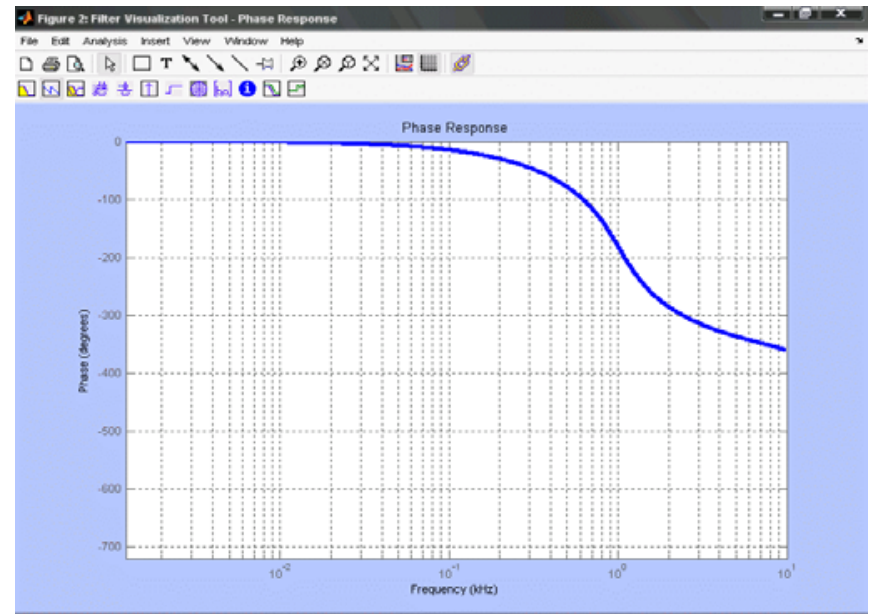

Fig.4. (b) Simulated responses of $4^{\text {th }}$ order Butterworth low-pass filter phase response in degree

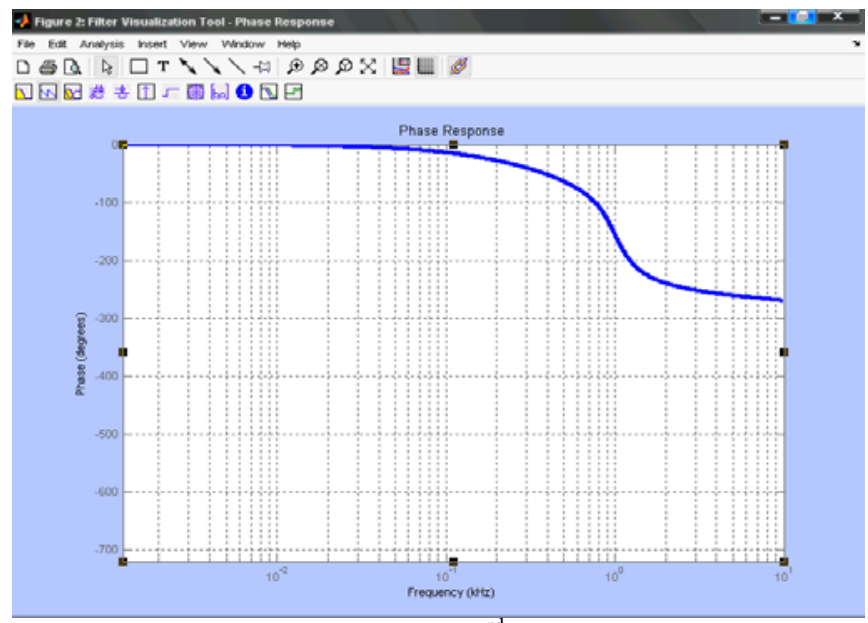

Fig.5. (b) simulated responses of $3^{\text {rd }}$ order Chebyshev type I low-pass filter phase response in degree

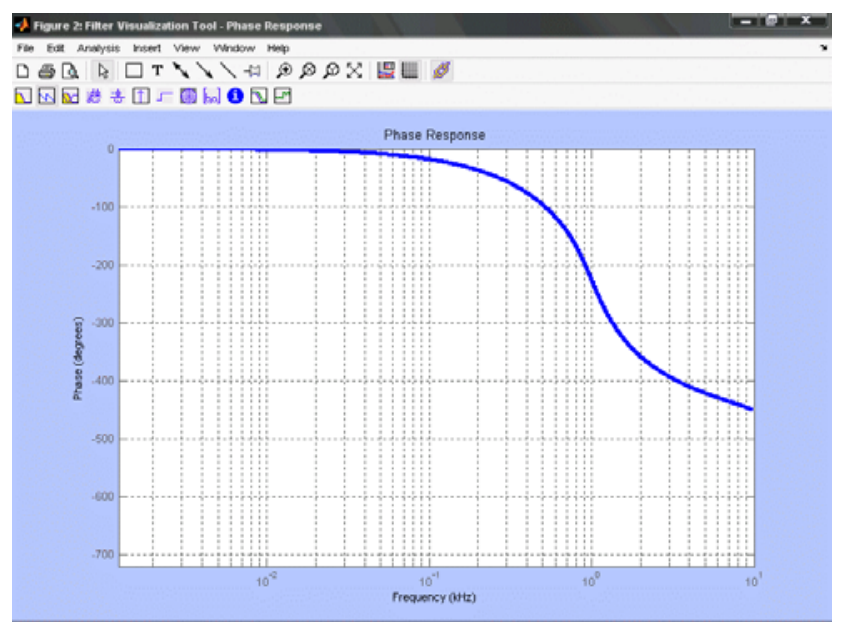

Fig.6. (b) simulated responses of $5^{\text {th }}$ order Bessel low-pass filter phase response in degree 


\section{Consideration}

As seen in previous subsection, the three filter types have different characteristics and thereby give different filter orders, Butterworth $4^{\text {th }}$ order, Chebyshev type I $3^{\text {rd }}$ order, and Bessel $5^{\text {th }}$ order. All three filters met requirements on attenuation in the stop band, but not on flat magnitude response requirements. The Bessel filter and Chebyshev type I look suitable but Butterworth met the requirement flat magnitude response best and is thereby decided to be implemented.

\section{IMPLEMENTATION BUTTERWORTH LOW-PASS \\ FILTER DESIGN}

A low-pass Butterworth filter of $4^{\text {th }}$ order using Sallenkey architecture is going to be used. When implementing a filter, the resistors and capacitors part of the Sallen-Key architecture must be calculated at first. Start the form writing out of the four poles below.

$$
\sigma_{p} \pm i \omega_{p}
$$

By using the buttap function of Matlab, the poles can be given as;

$$
\begin{gathered}
{[\mathrm{z}, \mathrm{p}, \mathrm{k}]=\operatorname{buttap}(4) ;} \\
\mathrm{P} \quad-0.9329+0.3827 \mathrm{i} \\
-0.9329-0.3827 \mathrm{i} \\
-0.3827+0.9329 \mathrm{i} \\
-0.3827-0.9328 \mathrm{i}
\end{gathered}
$$

Thus, Equation(1) can be rewritten as

Stage (1). $-0.9329 \pm 0.3827 \mathrm{i}$ and

Stage (2). $-0.3827 \pm 0.9329 \mathrm{i}$ respectively.

The quadratic function is

$$
N(s)=\left(s+\sigma_{p}-\omega_{p}\right) \cdot\left(s+\sigma_{p}+\omega_{p}\right)=s^{2}+2 s \sigma_{p}+\sigma_{p}^{2}+\omega_{p}^{2}
$$

Then, gives following quadratic functions on the four poles; for stage (1) $\mathrm{s}^{2}+1.8478 \mathrm{~s}+1$, for stage (2) $\mathrm{s}^{2}+0.7654 \mathrm{~s}+1$.

Quality values, Q are calculated according to Equation (3), for complex-pole pairs.

$$
Q=\frac{\sqrt{\mathrm{Re}^{2}+|\mathrm{Im}|^{2}}}{2 \mathrm{Re}}
$$

Where Re is the real part of the complex-pole pair, and Im is the imaginary part. The respective quality values of the complex-pole pairs and the single pole is 1.847 for stage (1) and 0.7654 for stage (2).

Next, a relationship between the resistors and capacitors need to the derived, which called $m$ and $n$. The quality value Q can be written as
$Q=\frac{\sqrt{m \cdot n}}{m+1}$

$Q^{2}(m+1)^{2}=m n \quad$ then, $m+2+\frac{1}{m}=\frac{n}{Q^{2}}$

Let $\left(\frac{n}{Q^{2}}-2\right)=x$

and now Equation( 4) can be written as

$m^{2}-x m+1=0$

The constant $\mathrm{n}$, which is the relationship between the two capacitors in the pole pair cases. It should be chosen with respect to values of the capacitors as exits to buy and also keep the resistors values to a few thousand ohms. That is why one of the roots in Equation (7) is thrown away.

The value $\mathrm{Q}$ influence the value $\mathrm{n}$, the higher $\mathrm{Q}$ the higher $\mathrm{n}$ should be used. For the stage (1). Choose $n=3.3$ and $\mathrm{Q}=0.54$ calculate $\mathrm{x}$ according to Equation (6) which give $\mathrm{x} \approx$ 9.32 .

The solution to Equation (7) is $\mathrm{m}_{1}=9.2$ and $\mathrm{m}_{2}=0.11$, where $m_{1}$ is thrown away.

Choose a value of the capacitor $\mathrm{C}_{1}=10 \mathrm{nF}$ and $\mathrm{C}_{2}=\mathrm{C}_{1} \cdot \mathrm{n}$ $=33 \mathrm{nF}$.For Sallen-Key architecture, $\mathrm{f}_{\text {pass }}$ is set to $1 \mathrm{kHz}$ and the frequency scaling factor denoted FSF, is calculated as

$F S F=\sqrt{\operatorname{Re}^{2}+|\operatorname{Im}|^{2}}$

Where Re real part of the complex-pole pair, and Im is the imaginary part. FSF $\approx 1$. From this the resistor values be calculated,

$R_{2}=\frac{1}{2 \pi \cdot C_{1} \cdot F S F \cdot f_{\text {pass }} \cdot \sqrt{m n}}$

which give $\mathrm{R}_{2}=26.4 \mathrm{k} \Omega$, and with the relationship $\mathrm{R}_{1}=\mathrm{R}_{2} \cdot \mathrm{m}=2.91 \mathrm{k} \Omega$.

For the stage (2), choose $n=\frac{100}{3.3}$ and $\mathrm{Q}=1.31$ calculate $\mathrm{x}$ according to Equation (6) which give $\mathrm{x} \approx 9.23$.

The solution to Equation ( 7 ) is $m_{1}=9.12$ and $m_{2}=0.1$, where $\mathrm{m}_{1}$ is thrown away. Choose the value of the capacitor $\mathrm{C}_{1}=6.8 \mathrm{nF}$ and $\mathrm{C}_{2}=100 \mathrm{nF}$. According to the Equation (8) and (9) get $\mathrm{R}_{2}=19.3 \mathrm{k} \Omega$ and then $\mathrm{R}_{1}=\mathrm{R}_{2} \cdot \mathrm{m}_{2}=1.9 \mathrm{k} \Omega$. 


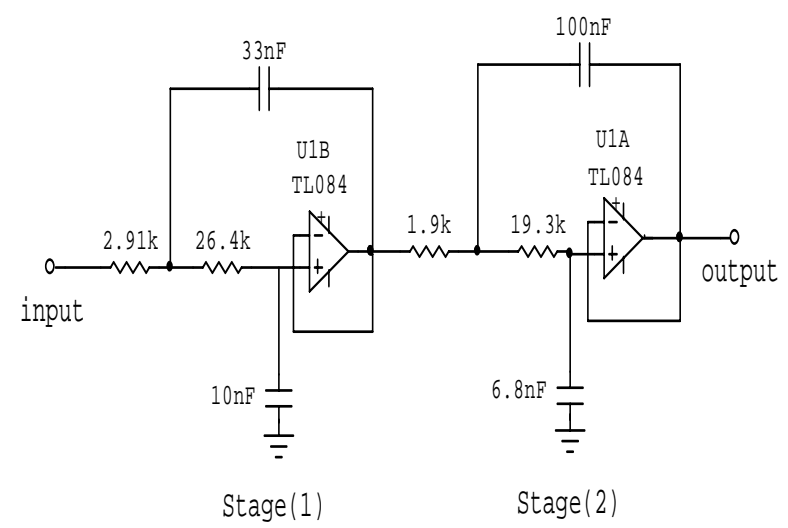

Fig.7. Schematic diagram of $4^{\text {th }}$ order Butterworth antialiasing filter
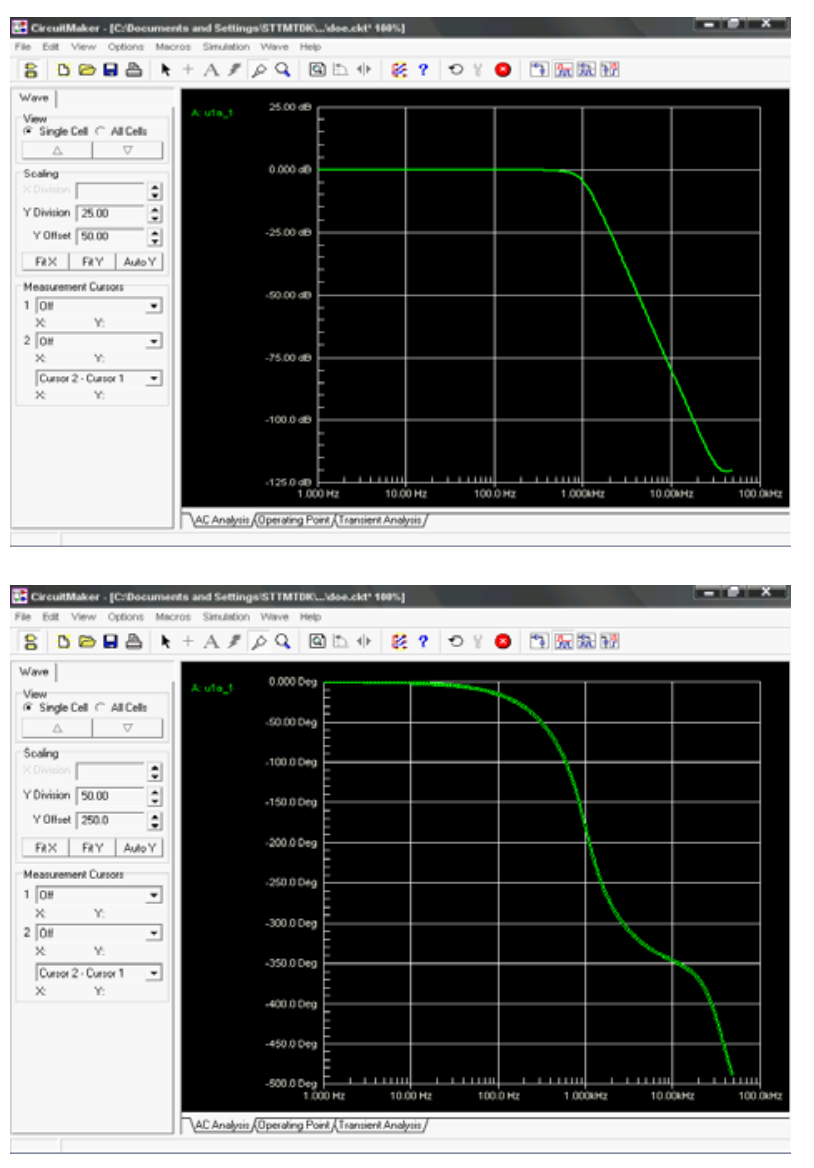

Fig.8. Simulated responses in Circuit Maker of $4^{\text {th }}$ order Butterworth low-pass filter; the upper plot shows the filter response in $\mathrm{dB}$ and the lower plot shows the phase function in degrees.

\section{DISCUSSION AND CONCLUSION}

Analog filtering is a critical portion of the data acquisition system. If an analog filter is not used, signals outside half of the sampling bandwidth of the A/D converter are aliased back into the signal path. Once a signal is aliased during the digitalization process, it is impossible to differentiate between noise with frequencies in band and out of band. The main purpose of this paper is to design active low-pass filter for data acquisition system. In this work, the filter is designed as two stages. The first stage is to determine which filter is used for this application and the second stage is chosen for Butterworth low-pass filter design and implementation. The circuit is designed using TL084 amplifier since this is good for data acquisition application. The simulated response from Circuit Maker of this circuit shown in Fig.8.For this circuit implementation, a $4^{\text {th }}$ order Butterworth is used with a cutoff frequency of 1 $\mathrm{kHz}$. Two Sallen-Key filters are used. This filter attenuates the pass band signal $-80 \mathrm{~dB}$ at $10 \mathrm{kHz}$. The simulated response is good and looks similiar with the simulated response in MATLAB. Therefore, the design filter is suitable for data acquisition application with anti-aliasing parameter.

\section{ACKNOWLEDGMENT}

The kindness and help of my supervisor Dr. Zaw Min Aung, Rector of Mandalay Technological University, Dr. Zaw Min Naing, pro-rector of Hlaing Tharyar Technological University and Dr. Yin Mon Myint, Assistance Professor Department of Electronic Engineering, Mandalay Technological University are gratefully acknowledged. Especially, I would like to express my special thank to my parents for their noble support and encouragement.

\section{REFERENCES}

[1] Analog Filter Design, Volkenburg, M.E. Van, Oxford University Press.

[2] Baker, Bonnie, "Using Operational Amplifiers for Analog Gain in Embedded System Design", AN682, Microchip Technologies, AN682, Microchip Technologies, Inc.

[3] Bonnie C.Baker," Anti-Aliasing, Analog Filters for Data Acquisition Systems “, AN699, Microchip Technology Inc.

[4] S.Winder, Analog and Digital Filter Design $2^{\text {nd }}$ ed. Woburn, MA: Newnes 2002.

[5] Active and Passive Analog Filter Design, An Introduction, Huelsman, Lawrence p, McGraw Hill, Inc.

[6] J.Karki. (2000.Oct) Active low-pass filter design. Texas Instrument. [Online].Available:

http://www-s.ti.com/sc/psheets/sloa049b/sloa049b.pdf. 